\title{
Mitigating Performance Degradation in Congested Sensor Networks
}

\author{
Raju Kumar, Student Member, IEEE, Riccardo Crepaldi, Student Member, IEEE, \\ Hosam Rowaihy, Student Member, IEEE, Albert F. Harris III, Member, IEEE, \\ Guohong Cao, Senior Member, IEEE, Michele Zorzi, Fellow, IEEE, and \\ Thomas F. La Porta, Fellow, IEEE
}

\begin{abstract}
Data generated in wireless sensor networks may not all be alike: some data may be more important than others and hence may have different delivery requirements. In this paper, we address differentiated data delivery in the presence of congestion in wireless sensor networks. We propose a class of algorithms that enforce differentiated routing based on the congested areas of a network and data priority. The basic protocol, called Congestion-Aware Routing (CAR), discovers the congested zone of the network that exists between high-priority data sources and the data sink and, using simple forwarding rules, dedicates this portion of the network to forwarding primarily high-priority traffic. Since CAR requires some overhead for establishing the high-priority routing zone, it is unsuitable for highly mobile data sources. To accommodate these, we define MAC-Enhanced CAR (MCAR), which includes MAC-layer enhancements and a protocol for forming high-priority paths on the fly for each burst of data. MCAR effectively handles the mobility of high-priority data sources, at the expense of degrading the performance of low-priority traffic. We present extensive simulation results for CAR and MCAR, and an implementation of MCAR on a 48-node testbed.
\end{abstract}

Index Terms-Wireless sensor networks, routing, congestion, differentiated service.

\section{INTRODUCTION}

SENSOR network deployments may include hundreds or $\mathcal{O}$ thousands of nodes. Since deploying such large-scale networks has a high cost, it is increasingly likely that sensors will be shared by multiple applications and gather various types of data: temperature, the presence of lethal chemical gases, audio and/or video feeds, etc. Therefore, data generated in a sensor network may not all be equally important.

With large deployment sizes, congestion becomes an important problem. Congestion may lead to indiscriminate dropping of data (i.e., high-priority (HP) packets may be dropped while low-priority (LP) packets are delivered). It also results in an increase in energy consumption to route packets that will be dropped downstream as links become saturated. As nodes along optimal routes are depleted of energy, only nonoptimal routes remain, further compounding the problem. To ensure that data with higher priority is received in the presence of congestion due to LP packets, differentiated service must be provided.

- R. Kumar, H. Rowaihy, G. Cao, and T.F. La Porta are with the Department of Computer Science and Engineering, The Pennsylvania State University, State College, PA 16802.

E-mail: \{rajukuma, rowaihy, gcao, tlp\}@cse.psu.edu.

- R. Crepaldi is with the Department of Computer Science, University of Illinois, Urbana-Champaign, IL 61801-2302. E-mail: rcrepal2@uiuc.edu.

- A.F. Harris III is with the Center for Remote Sensing of Ice Sheets (CReSIS), University of Kansas, Lawrence, KS 66045-7612.

E-mail: afh@cresis.ku.edu.

- M. Zorzi is with Department of Information Engineering, University of Padova, 35131 Padova, Italy. E-mail: zorzi@dei.unipd.it.

Manuscript received 6 Aug. 2007; revised 16 Nov. 2007; accepted 10 Jan. 2008; published online 28 Jan. 2008.

For information on obtaining reprints of this article, please send e-mail to: tmc@computer.org, and reference IEEECS Log Number TMC-2007-08-0232. Digital Object Identifier no. 10.1109/TMC.2008.20.
In this work, we are interested in congestion that results from excessive competition for the wireless medium. Existing schemes detect congestion while considering all data to be equally important. We characterize congestion as the degradation of service to HP data due to competing LP traffic. In this case, congestion detection is reduced to identifying competition for medium access between HP and LP traffic.

Congestion becomes worse when a particular area is generating data at a high rate. This may occur in deployments in which sensors in one area of interest are requested to gather and transmit data at a higher rate than others (similar to bursty convergecast [25]). In this case, routing dynamics can lead to congestion on specific paths. These paths are usually close to each other, which leads to an entire zone in the network facing congestion. We refer to this zone, essentially an extended hotspot, as the congestion zone (conzone).

In this paper, we examine data delivery issues in the presence of congestion. We propose the use of data prioritization and a differentiated routing protocol and/or a prioritized medium access scheme to mitigate its effects on HP traffic. We strive for a solution that accommodates both LP and HP traffic when the network is static or near static and enables fast recovery of LP traffic in networks with mobile HP data sources. Our solution uses a differentiated routing approach to effectively separate HP traffic from LP traffic in the sensor network. HP traffic has exclusive use of nodes along its shortest path to the sink, whereas LP traffic is routed over uncongested nodes in the network but may traverse longer paths.

Our contributions in this work are listed as follows:

- Design of Congestion-Aware Routing (CAR). CAR is a network-layer solution to provide differentiated 
service in congested sensor networks. CAR also prevents severe degradation of service to LP data by utilizing uncongested parts of the network.

- Design of MAC-Enhanced CAR (MCAR). MCAR is primarily a MAC-layer mechanism used in conjunction with routing to provide mobile and lightweight conzones to address sensor networks with mobile HP data sources and/or bursty HP traffic. Compared to CAR, MCAR has a smaller overhead but degrades the performance of LP data more aggressively.

We compare CAR and MCAR to an AODV scheme enhanced with priority queues (AODV $+P Q)$. Both CAR and MCAR lead to a significant increase in the successful packet delivery ratio of HP data and a clear decrease in the average delivery delay compared to AODV $+P Q$. CAR and MCAR also provide low jitter. Moreover, they use energy more uniformly in the deployment and reduce the energy consumed in the nodes that lie on the conzone, which leads to an increase in connectivity lifetime. In the presence of sufficient congestion, CAR also allows an appreciable amount of LP data to be delivered. We further show that, in the presence of mobile HP data sources, MCAR provides mobile conzones, which follow the HP traffic.

We also present the implementation of MCAR on our sensor network testbed. The implementation shows the feasibility of MAC-layer enhancements and differentiated routing on current hardware. We demonstrate that using an actual implementation, HP delivery rates similar to those seen in simulation can be achieved in a practical system.

The rest of this paper is organized as follows: Section 2 presents related work. Details of CAR and MCAR are presented in Section 3. Simulation details and results are presented in Section 4. Section 5 discusses our testbed implementation and results. Finally, Section 6 presents conclusions and future directions.

\section{Related Work}

An obvious solution to enhance service to HP data is to use priority queues to provide differentiated services (see [4], [15], and [25]). However, in such schemes, though HP packets get precedence over LP packets within a node, at the MAC layer, they still compete for a shared channel with LP traffic sent by surrounding nodes. As a result, without a routing scheme to address the impact of congestion and hotspots in the network, local solutions like priority queuing are not sufficient to provide adequate priority service to important data.

QoS in sensor networks has been the focus of current research (e.g., [4], [8], and [26]). SPEED [8] provides soft real-time guarantees for end-to-end traffic using feedback control and location awareness. It also concludes that local adaptation at the MAC layer alone is insufficient to address the problem of hotspots and that routing is essential to the solution. Akkaya and Younis [4] propose an energy-aware QoS routing protocol to support the delivery of real-time data in the presence of interfering non-real-time data by using multiple queues in each node in a cluster-based network; they do not consider the impact of congestion in the network and the interference that non-real-time traffic can cause to real-time data. Zhang et al. [26] propose a generic model for achieving multiple QoS objectives.

Degrading service to one type of data to provide better service to another has been used in schemes like RAP [15] and SWAN [3]. Similar to these works, we segregate data; however, instead of real-time delivery demands, we use data priority as the basis for our segregation.

Approaches like 802.11e [1] and other differentiated MAC schemes that assign higher priority to important data (e.g., VoIP for 802.11e) via MAC-layer mechanisms succeed at providing better service to HP data by assigning them preferential medium access. Funneling-MAC [2], proposed by Ahn et al., addresses the issue of increased traffic intensity in the proximity of a sink by using a schedulebased and contention-based MAC hybrid. As with data aggregation schemes like [16] and [21], it serves to delay the occurrence of congestion. Back pressure and rate limiting (also used in SPEED [8] and Fusion [10]) are essential to avoid situations where the network capacity is less than the amount of traffic being injected into the medium. Rangwala et al. [20] propose Interference-Aware Fair Rate Control (IFRC), which employs schemes to achieve fair and efficient rate limiting. It uses a tree rooted at each sink to route all data. When congestion occurs, the rates of the flows on the interfering trees are throttled. But, these schemes do not adopt differentiated routing. Also, in a large network that is under congestion in a constrained area, our approach leverages the large uncongested parts of the network that is often underutilized to deliver LP traffic.

RAP [15], SPEED [8], and MMSPEED [7] use velocitymonotonic scheduling. Applications assign an expected speed to each data packet, which is then ensured by these schemes. The speed that the application should assign to a packet if the network is congested is unclear. These schemes spread traffic around hotspots, but they do not give preference to HP data. In fact, if LP data has led to a hotspot in an area, routes for HP data that later enter the network will circumvent this hotspot. This will increase the number of hops over which this data has to be routed and increase the energy consumed in the network. In the worst case, no path for HP data may be found, and these packets will be dropped. Additionally, MMSPEED [7] achieves reliability by duplicating packets and routing them over different paths to the destination. Duplication of packets in congested networks may further precipitate congestion. Also, these schemes do not explicitly separate LP and $\mathrm{HP}$ traffic generated in the same area.

Our schemes are different from these schemes, because we use differentiated routing to provide the best possible service to HP data while trying to decrease the energy consumption in the conzone.

Congestion in sensor networks has been addressed in works like CODA [22], Fusion [10], and by Ee and Bajcsy [6]. Though these schemes take important steps to mitigate congestion in sensor networks, they treat all data equally. These schemes are complementary to the capability provided by CAR and MCAR. Similarly, our solutions do not preclude the use of priority queues, which can be added as a simple extension.

Existing work on congestion in sensor networks has two aspects: detection and mitigation. As mentioned earlier, we do not concern ourselves with congestion detection 


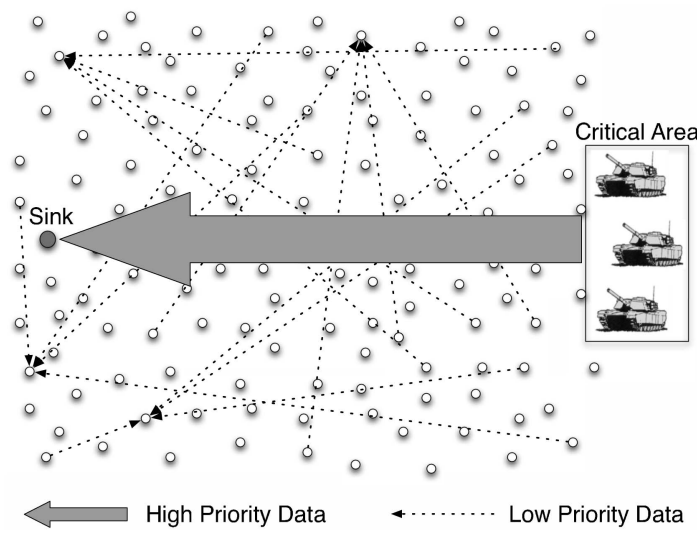

Fig. 1. A critical area of a sensor network may generate HP data at a high rate. This causes congestion in a part of the network exacerbated by the presence of LP data being routed in that area.

schemes in this work. Most mitigation schemes differ in how they invoke back pressure and rate limiting. Fusion's [10] mitigation scheme (other than back pressure and rate limiting) is assigning preferential medium access to parents in the routing tree. This assumes that all data in a network is destined to a single sink, which might not always be the case. In contrast, in our scenario, LP data can be sent from any node to any other node. As a result, Fusion's preferential MAC scheme is not applicable. Also, congestion in Fusion occurs due to the accumulation of packets close to the sink. In contrast, we address the degradation of performance of HP data delivery due to an extended hotspot in the network resulting from competition for medium access between LP and HP data. Also, Fusion does not do data differentiation based on priorities or provide differentiated routing.

\section{Congestion Aware Algorithms}

In Section 3.1, we introduce the network scenario and present an overview of our schemes, which are then detailed in Sections 3.2 and 3.3.

\subsection{Overview}

An example of the problem scenario that we consider is shown in Fig. 1. An important event occurs in one portion of the sensor field, which we call the critical area. This critical area will typically consist of multiple nodes. In such a scenario, there is a data processing center for collecting sensitive information from the critical area. Such data is assigned a higher priority than other data. There might also be several nodes collecting different types of LP information from other parts of the network. In the presence of this background LP traffic, without differentiating between the two priority classes, congestion will degrade the service provided to HP data. This may result in HP data being dropped or delayed so long that it is of no use to the data processing center. We refer to the area that contains the shortest paths from the critical area to the sink as the conzone. HP data would ideally traverse the conzone but will face competition for medium access due to LP traffic.

Our basic solution, called CAR, operates solely in the network layer. Packets are classified as HP or LP by the data sources, and nodes within a conzone only forward HP traffic. LP traffic is routed out of and/or around the conzone. In effect, we segment the network into two parts by using forwarding rules. One limitation with this system is that it requires some overhead to discover the conzone. While this overhead is reasonable, it may still be too heavyweight if the data source is moving often and the conzone is changing frequently or if the HP traffic is short lived. Hence, CAR is designed for static or nearly static networks with long-lived HP flows.

To address a mobile conzone (i.e., the conzone formed when sources of HP traffic are mobile) and/or bursty HP traffic, we define a MAC-layer-based protocol combined with routing to form conzones on the fly with each burst of data. This protocol handles mobility effectively but at the cost of drastically degrading the delivery of LP traffic, because there is no opportunity to establish alternate routes for such data. We call this second protocol MCAR. The combination of CAR and MCAR allows us to accommodate HP and LP traffic as best as possible, given the type of HP data source and the duration of HP traffic. For static sources, LP traffic finds alternate routes and suffers minor degradation using CAR. For networks with mobile nodes or bursty HP traffic, LP traffic is effectively interrupted and dropped when in contention with an HP source using MCAR.

\subsection{Congestion Aware Routing}

CAR comprises three steps: HP network formation, conzone discovery, and differentiated routing. The combination of these functions segments the network into on-conzone and off-conzone nodes. Only HP traffic is routed by on-conzone nodes. Note that the protocol specifically accommodates LP traffic, albeit with less efficient routes than HP traffic.

For the purposes of this discussion, we assume that there is one HP sink and a contiguous part of the network (critical area) that generates HP data in the presence of networkwide background LP traffic. We also assume that nodes are location aware (as in [8] and [14]) and densely deployed with uniform distribution.

Since nodes in the scenario in Fig. 1 send all HP data to a single sink, tree-based routing, with the HP sink being the root, is most appropriate. However, Hull et al. [10] show that tree-based routing schemes suffer from congestion, especially if the number of messages generated at the leaves is high. This problem becomes even worse when we have a mixture of LP and HP traffic traveling through the network. Therefore, even when the rate of HP data is relatively low, the background noise created by LP traffic will create a conzone that spans the network from the critical area to the HP sink. Due to this congestion, service provided to HP data may degrade, and nodes within this area may die sooner than others, leading to only suboptimal paths being available for HP data, or a network partition may result, isolating the sink from the critical area.

If a standard ad hoc routing scheme (e.g., AODV [18] or DSR [13]) is used to route the burst of HP data instead of the tree-based routing scheme, congestion occurs. Fig. 2 shows the conzone that is formed when AODV is used for routing all data in a deployment of 120 nodes. There is one HP sink and two LP sinks, as shown in the figure. Only critical area nodes send HP data, while all other nodes in the network send LP data to either of the LP sinks. We do not show a similar figure for Directed Diffusion [12] (using 


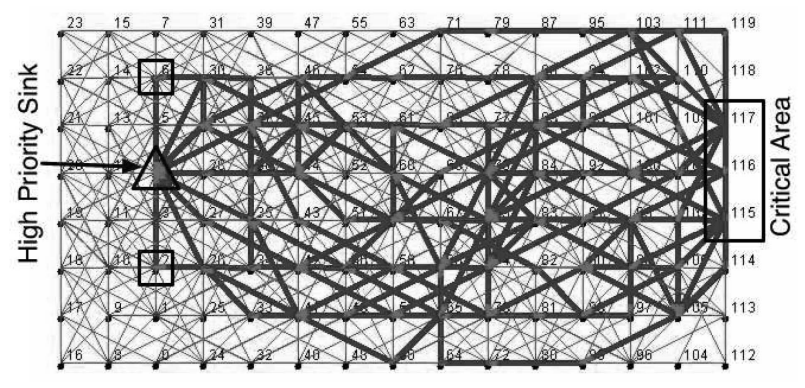

Fig. 2. Presence of congestion with AODV routing in a network subjected to HP data rate of 30 packets/sec (pps) and background LP traffic rate of $0.5 \mathrm{pps}$. Thin lines represent LP traffic, while thick lines represent HP traffic.

the One-Phase Pull Filter), because the control overhead of the initial flooding required with such a large number of data sources of LP and HP traffic was prohibitive and led to no HP data to be delivered.

We now present the algorithms used by CAR to build $\mathrm{HP}$ routing networks, to perform dynamic conzone discovery, and to provide differentiated routing. This is followed by the description of two enhancements of the basic CAR.

\subsubsection{High-Priority Routing Network Formation}

After the deployment of sensor nodes, the HP data collection center (the sink) initiates the process of building the HP routing network (HiNet). This network covers all nodes, because at the time of deployment, the sink will usually have no information on the whereabouts of the critical area nodes. Also, based on the locations of events that can occur during the lifetime of the network, different nodes may constitute the critical area.

Since all HP data is destined to a single sink, the HiNet is based on a minimum distance spanning tree rooted at the sink. As with TAG [16], this structure ensures that all nodes have shortest path routes to the sink. However, instead of every node having a single parent, as in other tree-based schemes, we allow nodes to have multiple parents. A node that has multiple neighbors with depths (the number of hops to the sink) less than its own considers them all as parents (see Fig. 3). We leverage this property to support multipath forwarding, thus providing load balancing and making the routing network more resilient to failures.

We now consider the HiNet formation process. Once the sink discovers its neighbors, it broadcasts a "Build HiNet" message (containing the $I D$ and depth of the node) asking all nodes in the network to organize as a graph. Once a neighboring node hears this message, it checks if it has already joined the HiNet (i.e., if it knows its depth); if not, it sets its depth to one plus the depth in the message received and sets the source of the message as a parent. This node then rebroadcasts the Build HiNet message, with its own ID and depth. If a node is already a member of the graph, it checks the depth in the message, and if that depth is one less than its own, then the source of the message is added as a parent. In this case, the message is not rebroadcast.

If a node receives a Build HiNet message with a depth value less than that of its parent's depth, it updates its own value to the received value, plus one. It then removes all current parents and adds the source of the message as a

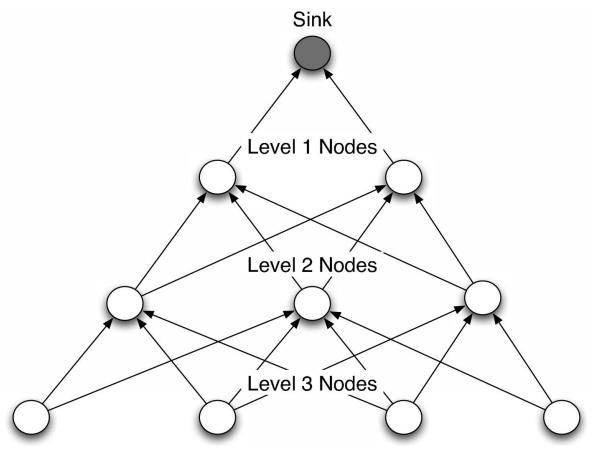

Fig. 3. In a dense deployment, multiple nodes can be parents of a node. Each parent lies on a different shortest path route to the sink. This structure is used for shortest multipath routing.

new parent. Finally, the Build HiNet message is rebroadcast with the new depth value. In this fashion, the Build HiNet message is sent down the network until all nodes become part of the graph. Similar to TAG [16], the Build HiNet message can be periodically broadcast to maintain the topology and adapt to changes caused by the failure or addition of nodes.

\subsubsection{Dynamic Conzone Discovery}

Nodes discover if they are on the conzone by using the conzone discovery mechanism. After building the HiNet, the next task is to dynamically discover the conzone. The conzone is formed when one area is generating HP data. We refer to this area as the critical area. This conzone discovery is done dynamically, because the critical area can change during the lifetime of the deployment and is triggered when an area starts generating HP data.

The conzone can be discovered and destroyed either from the critical area nodes to the sink or vice versa. The conzone discovery algorithms allow nodes, in a distributed fashion, to determine if they are on a potentially congested path between the critical area and the sink. If they are, they mark themselves as "on conzone." The conzone discovery schemes are summarized in Fig. 4.

For brevity, we only present conzone discovery from the critical area to the sink in detail. In this case, critical area nodes detect an event that triggers discovery. A conzone must be then discovered from that neighborhood to the sink for the delivery of HP data. To do this, critical area nodes broadcast "discover conzone to sink" (ToSink) messages. This message includes the ID of the source and its depth and is overheard by all neighbors. The depth is included here to ensure that nodes do not respond to the ToSink messages heard from their parents. When a node hears more than $\alpha$ distinct ToSink messages coming from its children, it marks itself as on conzone and propagates a single ToSink message. This message is overheard by neighbors who mark this neighbor as being on the conzone in their neighborhood table. In our scheme, this threshold $\alpha$ is a linear function of the neighborhood size (i.e., the number of nodes within the communication range) and of the depth of the node in the HiNet, as shown in (1). For node $x$ with depth $d_{x}$ and neighborhood size $n_{x}$, we have

$$
\alpha_{x}=\beta_{d_{x}} \cdot d_{x} \cdot n_{x}
$$




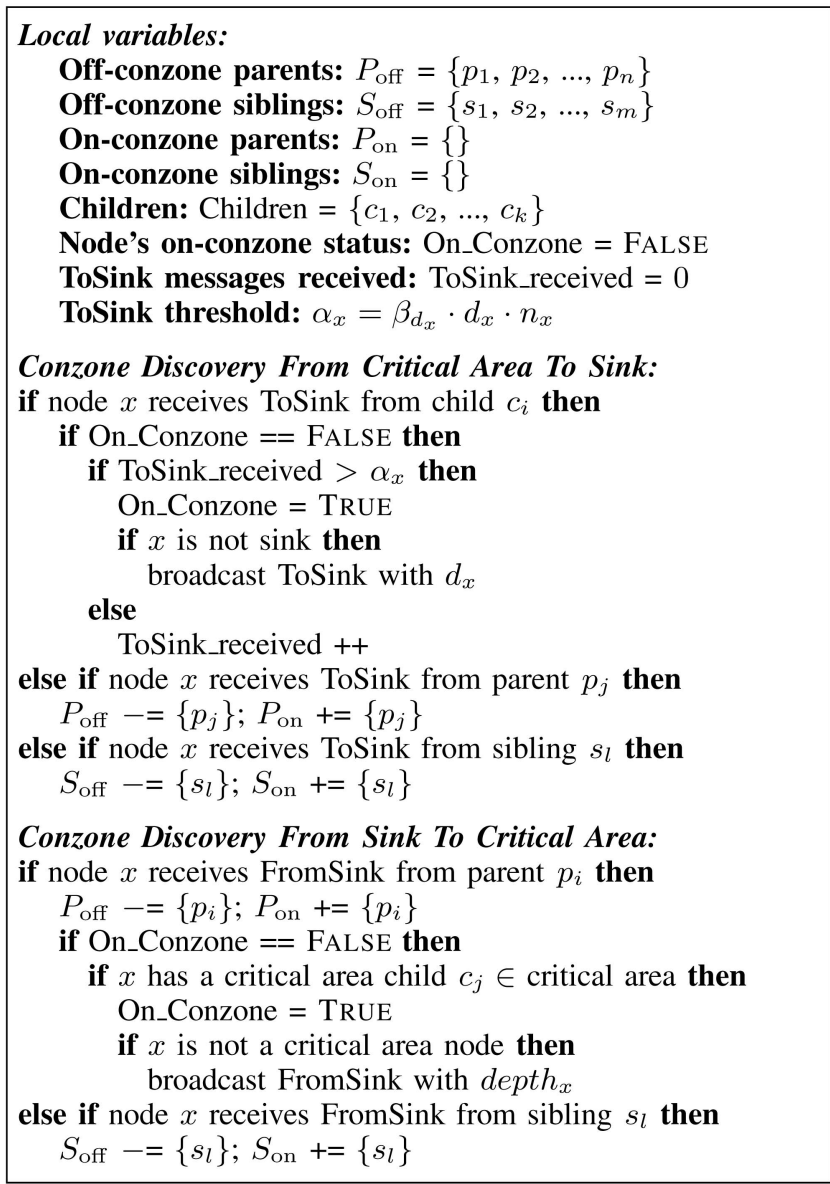

Fig. 4. Conzone discovery algorithms in CAR for node $x$.

Since the depth and neighborhood size can vary for different nodes, $\alpha$ is set accordingly. Setting $\beta$ correctly for different depths ensures that the conzone is of an appropriate width. As $\beta$ becomes smaller, the conzone becomes wider. Depth must also be taken into account, because if $\alpha$ is the same for different depths, the conzone will become very narrow as it approaches the sink. Note that due to the assumption of uniform deployments, the neighborhood size is related to the number of children by a constant factor. Hence, (1) can be adapted to use the number of children, but we use the neighborhood size instead.

An important goal of the conzone discovery algorithm is to split the parents and siblings (nodes with the same depth) in the HiNet into on-conzone and off-conzone neighbors. Initially, all parents and siblings are marked as off conzone. Since a node will forward a ToSink message only if it becomes on conzone, when a node hears such a broadcast from its parent(s) or sibling(s), it marks that neighbor as on conzone.

Since the presence of a conzone leads to suboptimal routing for LP data due to on-conzone nodes being dedicated to serving HP data, after the HP stream comes to an end, the conzone is destroyed by flooding a "destroy conzone" message in the conzone.

\subsubsection{Differentiated Routing}

Once the conzone is discovered, HP data is routed in the conzone, and LP data is routed off the conzone. Since the

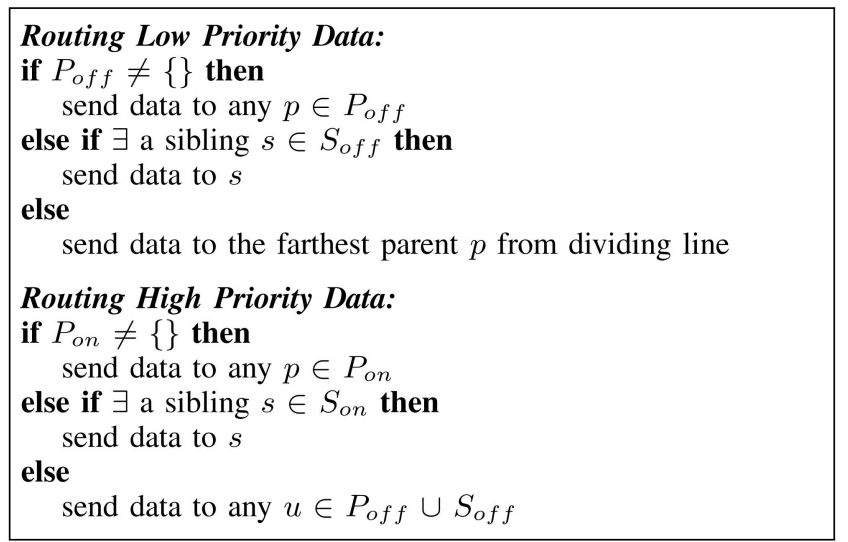

Fig. 5. Routing algorithm for CAR for LP and HP data inside the conzone.

critical area is part of the conzone, all HP data will be generated inside the conzone. Hence, the routing of HP data is simple: a node always forwards the data to one of its onconzone parents. This parent is chosen randomly from the on-conzone parent list to balance the load among them. If, for some reason, the links to all parents are broken, for example, because of node failures, the node will forward the data to a sibling that is on the conzone. If that is impossible, it will forward the data to any of its neighbors, hoping that it can return to an on-conzone node.

LP data generated inside the conzone is routed out using the following approach. When an on-conzone node gets an LP message, it forwards it to an off-conzone parent, if there are any. Otherwise, the LP data is forwarded to an offconzone sibling. If there are no parents or siblings that are off conzone, we resort to the following method. After discovering the conzone, the sink sends a message through the conzone, which contains the coordinates of a line that cuts the conzone in half. This line connects the sink to the center of the critical area. Using this information and its own coordinates, a node can determine on which half of the conzone it lies and hence routes LP data to the parent that is closest to the conzone boundary, i.e., farthest from the line. With the assumption of uniform deployment density, this ensures that all LP data generated inside the conzone is routed out efficiently and along the shortest path.

The routing scheme described above is highly efficient for LP traffic flowing in the same direction as the HP traffic. Though it is not optimal for LP traffic flowing in different directions, it will still correctly deliver the data while keeping the routing out cost low.

It is important to note here that to keep the routing overhead low, LP routing decisions inside the conzone are static. So, once a node decides to which neighbor it is going to forward LP data, it uses the same neighbor for all LP packets. If that neighbor fails, an alternative must be found using the same scheme. In-conzone routing for both LP and HP data is summarized in Fig. 5.

LP data generated outside the conzone or routed out of the conzone has to be routed to the appropriate LP sink without using the conzone nodes. Hence, routing LP data outside the conzone can use any of the known routing schemes such as AODV, with modifications to prevent LP data from being routed from an off-conzone node into 
the conzone. We used AODV in the off-conzone nodes to route LP data, with the modification that the on-conzone nodes do not propagate route request or reply messages for LP data. Using this modified routing scheme, LP data generated outside or routed out of the conzone is routed to its destination via off-conzone nodes only.

\subsubsection{Enhancements}

In CAR, LP data generated inside the conzone requires the conzone nodes to dedicate some of their resources to route such data out of the conzone. As an enhancement to better serve HP data, on-conzone nodes stop generating or forwarding any LP data. We call this enhancement CAR+.

Due to the shared nature of the wireless channel, HP messages can be dropped by the critical area nodes themselves due to collisions with other LP data from neighboring nodes. This is especially true if the amount of LP traffic surrounding the critical area is large. As the second improvement, we disable generating and forwarding of LP data in all nodes that are within the communication range of any critical area node. Since nodes know their neighbors and their status, once a node discovers that one of its neighbors is on the critical area, it disables generating and forwarding of any LP data. We call this enhancement CAR++.

\subsection{MAC-Enhanced Congestion Aware Routing}

In this section, we present MCAR, a combined MAC and routing scheme designed to support situations in which critical events may move or the sensors generating HP data may move. Though conzone discovery is dynamic in CAR, the overhead required to maintain the HiNet in a dynamic environment may be prohibitive. As a result, we use a lightweight dynamic differentiated routing mechanism to accommodate mobile data sources. MCAR is based on MAC-layer enhancements that enable the formation of a conzone on the fly with each burst of data. The trade-off is that it effectively preempts the flow of LP data, thereby seriously degrading its service.

Unlike CAR, MCAR does not form an HP network. Instead, HP paths are dynamically created, since the sources (or the sinks) are expected to be mobile. Thus, MCAR discovers the conzone while discovering the paths from HP sources to the sink.

The enhanced MAC-layer of MCAR uses an RTS/CTS protocol that is augmented to carry information about the priority level of the data being transferred. Each RTS and CTS packet is tagged with a priority level. During channel contention, if a node has HP data to send and overhears an LP RTS, it jams the channel with an HP CTS, causing nodes forwarding LP data to back off. Furthermore, if a node with LP data overhears an HP RTS or CTS, it will back off the channel, as described in the following section.

Though 802.11e [1] is similar to MCAR in that they both prioritize access to the medium, the prioritized RTS/CTS messages in highly congested networks may be dropped. 802.11e's policy of guarding every transmission with an RTS/CTS exchange leads to a prohibitive overhead. Woo and Culler [23] state that RTS/CTS exchange imposes an overhead of up to 40 percent. The extent of overhead experienced depends on the relative size of the RTS/CTS packets and the data packets. In sensor networks, data packet sizes are

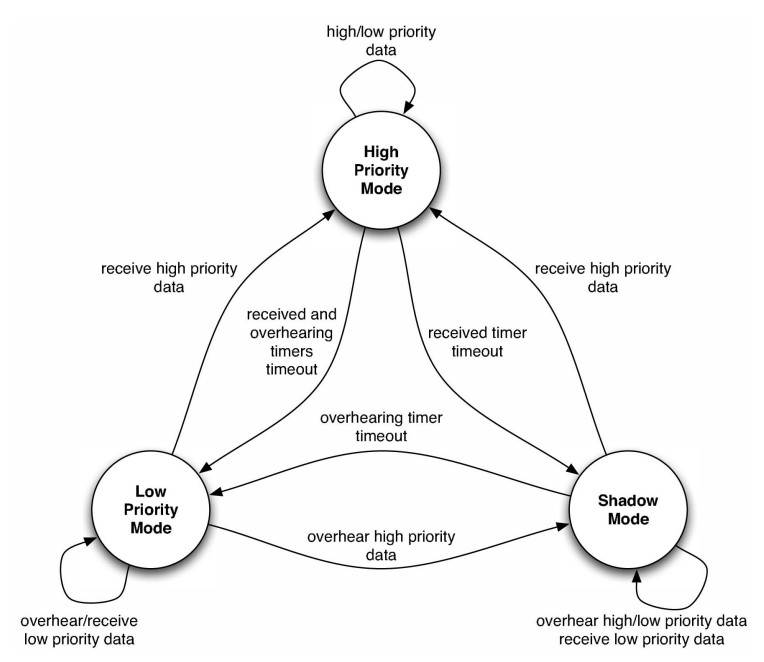

Fig. 6. MCAR state machine.

not large enough to justify the cost of RTS/CTS exchange to guard every packet. Hence, 802.11e is unsuitable for sensor networks. MCAR uses a silencing mechanism that does not require preempting all LP data transmissions in the neighborhood for each HP data to be sent. Rather, MCAR silences the conzone and its neighborhood during route discovery and/or maintenance.

Though the cost of an RTS/CTS exchange for each data packet may be considerable for a sensor network, even S-MAC [24], a widely used MAC scheme for sensor networks, uses one RTS/CTS exchange for a collection of message fragments. Similarly, the cost of RTS/CTS imposed by MCAR is not prohibitive, since it uses these RTS/CTS packets only during the route discovery/maintenance phase. Hence, the scalability of the RTS/CTS overhead for MCAR is not an issue.

In MCAR, nodes discover if they are on the conzone by using the conzone discovery explained in the following. Like CAR, this conzone discovery is triggered when an area starts generating HP data. For the conzone to be discovered dynamically, MCAR uses two timers to regulate when a node decides it is no longer part of the HP path. One timer, called the overhearing timer, monitors how long it has been since the last HP packet was heard. This timer is used to control nodes in the communication range of the conzone but that are not necessarily involved in forwarding the packets. The overhearing timer is reset any time an $\mathrm{HP}$ packet is overheard or any time an HP packet is received (since nodes involved in forwarding packets are clearly within the communication range of nodes transmitting those packets). The second timer, called the received timer, controls nodes either generating or forwarding HP data.

In MCAR, each node in the network can be in one of three states, dictating whether it is a part of the conzone or not or within the communication range of the conzone but not a part of it (see Fig. 6). This last mode creates a shadow area that separates HP traffic from LP traffic.

\subsubsection{State Machine}

The node state machine used by MCAR to support differentiated routing based on MAC-layer enhancements is shown in Fig. 6. 
LP mode. In this mode, nodes forward LP data. All nodes in the network are initially in the LP mode. Upon receiving or overhearing an LP packet, nodes remain in the LP mode and, if appropriate, forward any data. If a node in the LP mode overhears an HP packet, it transitions to the shadow mode. Finally, upon receiving an HP event that needs to be forwarded (either because it sensed an HP event or because it was chosen as the next hop toward the sink), a node transitions to the HP mode.

HP mode. Nodes in the path of HP data are in the HP mode. Upon transitioning to this state, the node sets two timers: a received timer and an overhearing timer. The values for these timers should be on the order of twice the expected interarrival delay of HP data.

If a node in this mode receives an HP transmission, it begins channel contention by using our modified RTS/CTS protocol and forwards the data. It resets its received and overhearing timers and remains in the HP mode. Upon overhearing HP data, the node resets its overhearing timer only and stays in the HP mode.

If a node in the HP mode overhears or receives an LP RTS, it sends a jamming HP CTS to clear the channel of LP data and to announce the existence of an HP path and stays in the HP mode.

If the received timer expires, the node transitions to the shadow mode, maintaining the value of its overhearing timer. While this is the normal exit out of the HP mode, if both the received timer and overhearing timer expire at the same time, the node transitions back to the LP mode.

Shadow mode. Nodes in this state are within the communication range of HP traffic but not on a forwarding path. Nodes in this state suppress LP traffic, thus preventing it from interfering with HP traffic in the network. Upon overhearing an HP packet, the node resets its overhearing timer and stays in this state. A node transitions to the $\mathrm{HP}$ mode upon receiving an HP packet itself.

If a node in the shadow mode overhears an LP packet, it stays in the shadow mode and takes no action. If the node is the intended recipient of the LP data, it silently discards the packet and stays in the shadow mode. It should be pointed out that this is an aggressive action to maximize the service given to HP data. Finally, if the overhearing timer expires, the node transitions to the LP mode.

\subsubsection{Routing}

Route discovery is performed dynamically at the time of HP event detection. Essentially, MCAR performs on-demand route discovery similar to schemes like AODV. The route discovery and reply packets are marked according to the priority of impending data, causing nodes along the route for HP data to transition to the HP mode. Once the route is built, HP data flows along this path. In the event of a route break due to node failure or mobility, route recovery is performed, again using HP control packets. Nodes on segments of the old route will transition back to the LP mode as their timers expire, and LP flows that were not forwarded can now be transmitted.

Only nodes in the LP mode forward LP data, including any LP route requests. The routing of this data can be performed using any routing mechanism and is orthogonal to the routing mechanisms used by MCAR. Nodes in the
TABLE 1

Summary of Schemes

\begin{tabular}{|l|l|}
\hline Scheme & Summary \\
\hline CAR & $\begin{array}{l}\text { For static or nearly-static conzone and long-lived high } \\
\text { priority flows }\end{array}$ \\
\hline CAR+ & Conzone nodes drop all low priority data \\
\hline CAR++ & $\begin{array}{l}\text { Conzone nodes and neighbors of critical area drop all } \\
\text { low priority data }\end{array}$ \\
\hline MCAR & $\begin{array}{l}\text { For mobile high priority data sources or short-lived } \\
\text { high priority flows }\end{array}$ \\
\hline
\end{tabular}

HP or the shadow mode drop LP data. Hence, there is no need to route LP data out of the HP zone in MCAR. As a result, MCAR is more aggressive in dropping LP data and eliminates all competition for the shared channel among the LP and HP packets. This is one of the trade-offs between CAR and MCAR.

Although both schemes support HP data delivery, CAR is able to route LP traffic out of the conzone, while MCAR cannot. CAR requires the formation of the HiNet, which incurs higher overhead than the dynamic path establishment of MCAR. CAR is more permissive of LP traffic than MCAR: it allows nodes that would be in the shadow mode in MCAR to forward LP data. MCAR, on the other hand, performs more similarly to $\mathrm{CAR}++$ in this respect, limiting the use of nodes in the conzone to only HP data. Section 4 quantifies these trade-offs through simulation studies.

\subsection{Congestion Aware Routing and Mobility}

In many applications, sensor networks are static or are characterized by low mobility. While MCAR is designed for networks with high mobility, CAR is suitable for very low mobility applications. Though MCAR can be applied to scenarios with low mobility, it aggressively degrades service to LP traffic. CAR can be adapted to avoid such degradation of service to LP data. Each component of CAR-HP routing network formation, conzone discovery, and differentiated routing — can be modified to handle low mobility. Every time a neighbor moves, it needs to discover its new parents and children in the HP network. This can be achieved by a periodic beacon message flood that organizes the network as a tree. As long as a conzone node stays in the conzone or an off-conzone node stays off the conzone, no change is required. If a conzone node moves to an off-conzone area, we can use a time-out mechanism similar to the one in MCAR to switch it to the off-conzone mode. If an off-conzone node moves to the conzone, it can switch itself to conzone if a sufficient number of its new children transmit HP packets. Though we do not verify the efficacy of CAR under such low mobility cases, our results for the static scenarios give us confidence in these expectations, as described in the following.

\section{Performance Evaluation}

In this section, we describe our simulation setups used to test CAR and MCAR and discuss the results in detail. Table 1 provides a brief summary of our proposed schemes.

Since our implementation testbed consists of only 48 nodes, we use larger setups in simulations to gather insights about CAR-based schemes and MCAR. Hence, we present extensive simulation results in this section, which 


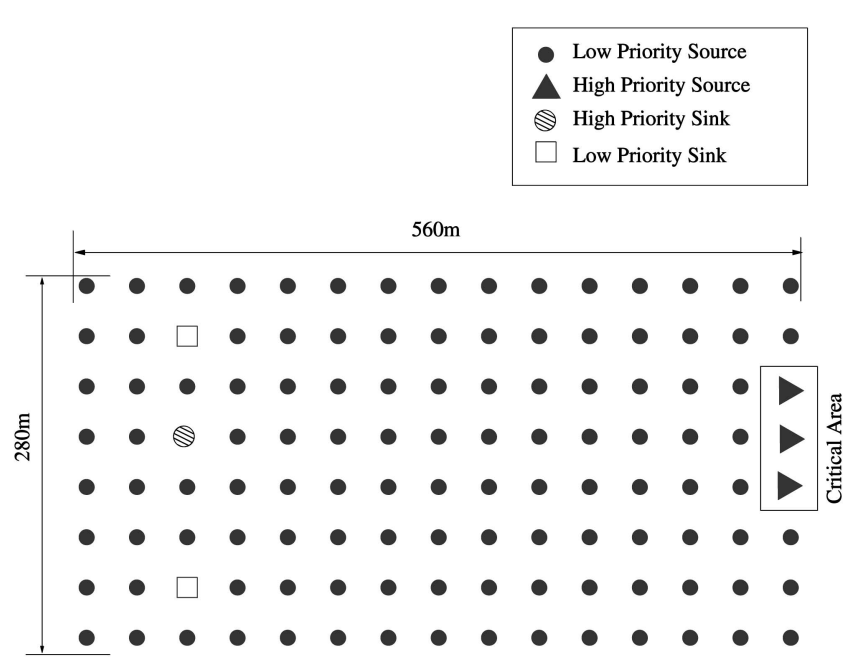

Fig. 7. Simulation scenario.

are complemented by implementation results in Section 5 to complete the picture.

\subsection{Simulation Setup}

The simulations were conducted in NS-2 [17], with a deployment area of $560 \mathrm{~m} \times 280 \mathrm{~m}$. In this area, 120 nodes are placed in a $15 \times 8$ grid, as shown in Fig. 7, with the separation between neighboring nodes along both axes being $40 \mathrm{~m}$. Note that we use grids as deployments in this paper to emulate uniformly dense deployments and such grids are not a requirement of our algorithms. As long as the neighborhood relationships are similar, the results will not differ significantly from those presented in this paper.

Two LP sinks receive all LP data, while a single sink receives all HP data. Three nodes form the critical area and send HP data. The rest of the nodes, other than the three sinks and the three critical area nodes, send LP data to either LP sink (see Fig. 7). This LP data serves as the background traffic in our simulations. Note that the HP sources in our simulations were placed at the edge of the deployment to get a sufficient number of hops from them to the HP sink. In a large deployment of hundreds of nodes, these HP sources need not be at the edge of the deployment. Results were recorded when the system reached a steady state. CAR uses AODV to route LP data outside the conzone, with a modification to ensure that offconzone nodes do not route such data into the conzone.

The IEEE 802.11 is used as the MAC layer operating at 11 Mbps. 802.11 is a CSMA/CA MAC layer that uses RTS/ CTS to avoid the hidden terminal problem. Sensor network MAC schemes like S-MAC [24] and B-MAC [19] employ CSMA/CA and cut down the overhead of RTS/CTS. However, in dense networks that are under high congestion, these schemes will need to use RTS/CTS for each data packet to avoid the hidden terminal problem. Hence, 802.11 is a reasonable approximation of S-MAC [24] under congested conditions. Actually, the results in [24] show that a node that forwards sufficient traffic uses less energy with 802.11 than with S-MAC. Since nodes in the congested part of the network will often forward a significant amount of traffic, 802.11 is more energy efficient than S-MAC for congested networks.
We compare CAR (and its improvements) and MCAR to AODV and to an enhanced version of AODV that we implemented, that is, $\mathrm{AODV}+\mathrm{PQ}$. $\mathrm{AODV}+\mathrm{PQ}$ maintains two queues at each node. The first is an HP queue. Messages in this queue are transmitted if present. The second queue is an LP queue. When the HP queue is empty, messages from this queue are transmitted. This policy provides absolute privilege to $\mathrm{HP}$ data within a node. $\mathrm{AODV}+\mathrm{PQ}$ is a simple generalization of priority-queuebased schemes such as the ones used in [4], [15], and [25].

We also generated results for DSR [13] and Directed Diffusion [12] but do not present them here. In our environment of large multihop networks, DSR fails to route any HP data successfully. DSR is intended to work over networks with a small number of hops, as reported in [5]. Similarly, Directed Diffusion was unable to route any HP data successfully due to the large control overhead involved in the initial flooding that is required to set up the data paths. One-Phase Pull Filter was used in the simulations, and though it is expected to route LP packets successfully, our simulations showed that as the number of senders in the deployment was increased beyond 10, Directed Diffusion failed to route any data. As with DSR, Directed Diffusion is not intended for such applications. It was mainly designed to work in cases where the number of sinks and senders is small.

We do not compare our work to solutions that propose rate limiting, back pressure, or throttling of senders as mitigation schemes (Fusion [10] and CODA [22]), because they treat all data equally and do not utilize the uncongested parts of the network to deliver LP data. Also, Fusion's prioritized medium access scheme is designed for situations in which all data in the network is routed to the same sink. This might not always be true, e.g., three sinks are used in an experiment in CODA [22]. We also do not compare our schemes with SPEED [8], because its Stateless Nondeterministic Geographic Forwarding scheme is agnostic of data priority. If LP data leads to an extended hotspot in the network, HP flows that later enter the network will be forced to circumvent the hotspot. In contrast, we provide shortest paths for HP flows and force LP data to circumnavigate the conzone. In the worst case, SPEED will drop HP packets due to the unavailability of a suitable downstream node if all neighbors are sufficiently congested with LP traffic. These are the scenarios in which we try to prevent degradation of service to HP data.

A routing scheme has different aspects: route formation overhead, the quality of routes, route maintenance overhead, etc. For this work, only the quality of routes is vital. The rest of the aspects of a routing scheme are not central to the problem of performance in congested networks. Since AODV uses shortest paths, we selected the same. Also, AODV is a widely used routing scheme, and several other routing schemes are based on it. Hence, like SPEED, we compare the performance of our schemes to AODV.

In our simulations, CAR builds the conzone from the critical area to the sink. Nodes are added to the conzone if they receive at least $\alpha=2$ ToSink messages. For example, with a transmission range of $130 \mathrm{~m}$, the neighborhood size of a node away from the edge of the deployment is equal to 36 . For a node at depth $3, \beta_{3}$ is set to 0.018 . For nodes with 


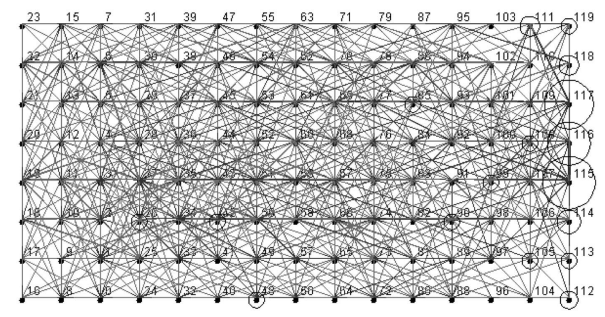

(a)

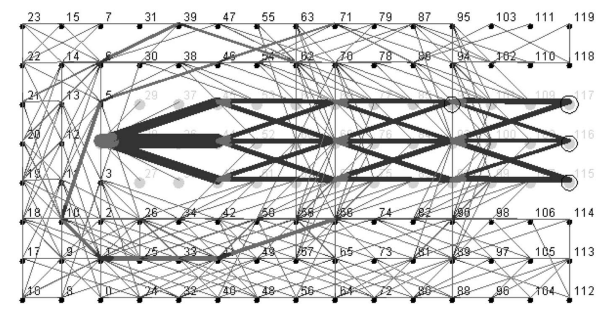

(b)

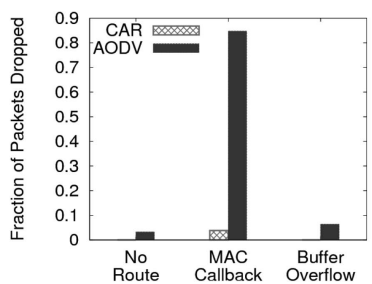

(c)

Fig. 8. Routing views for all data and packet drop analysis for HP packets for AODV and CAR for range $=130 \mathrm{~m}$, LP data rate $=0.5 \mathrm{pps}$, and HP data rate $=30$ pps. (a) Routing view for AODV. (b) Routing view for CAR. (c) HP packet drop analysis.

depth $2, \beta_{2}$ is set to 0.027 , and so on. MCAR uses the algorithms described in Section 3.3 to build its conzones.

We now provide a high-level comparison of CAR with AODV. Figs. $8 \mathrm{a}$ and $8 \mathrm{~b}$ depict the routing of LP and HP packets by AODV and CAR. CAR+, CAR++, and MCAR look similar to CAR, as they all route only HP traffic in the conzone. Lightly shaded edges denote LP data, while heavily shaded edges denote HP data. The thickness of edges is directly proportional to the number of packets routed over them. A circle around a node denotes that the node has dropped HP packets. The radius of the circle is directly proportional to the number of HP packets dropped. Also, the larger nodes in Fig. $8 \mathrm{~b}$ denote the nodes that belong to the discovered conzone.

In Fig. 8a, we observe that AODV routes both LP and $\mathrm{HP}$ data along the same paths and these paths may not be the shortest possible. As a result, many HP packets are dropped at the critical area nodes themselves. Additionally, in CAR, a conzone is formed (this can be observed in Fig. $8 \mathrm{~b}$ ). The set of shortest paths from the critical area to the sink routes all HP data, while the rest of the network routes LP data. LP data generated inside the conzone for CAR is effectively routed out using the minimum number of hops inside the conzone. It can be seen in Fig. $8 \mathrm{~b}$ that CAR effectively performs differentiated and multipath routing and successfully routes routed-out or off-conzone generated LP data around the conzone.

In the examples in Figs. 8a and 8b, AODV routes only 5.7 percent of HP data successfully, while CAR delivers 96.2 percent of such data. The most prominent reason for AODV dropping packets, based on our analysis shown in Fig. $8 c$, is that the MAC layer fails to route a packet after several retransmission attempts (MAC Callback). This is due to congestion, which makes it difficult for a node to capture the channel to transmit data. Also, while AODV delivers 78 percent of LP data, CAR delivers 89 percent of such data.

Note that in all simulations, the queue size at each node was set to 1,000 packets, while the size of the packets was 50 bytes. This requires around a 50-Kbyte memory space, which is easily available in different motes (e.g., EyesIFXv2 [11] motes have a 512-Kbyte serial EPROM, and MicaZ motes have a 512-Kbyte measurement flash). Note that RAP [15] uses a queue size of 300 packets. Since the queues in nodes are almost always nonempty during congestion, the rate of "MAC Callback" errors will remain the same. AODV benefits more than CAR-based schemes and MCAR with a large queue size, because it drops more HP packets due to buffer overflow (see Fig. 8c). With a small queue,
AODV will drop more data due to buffer overflow, while CAR-based schemes and MCAR will drop much less data.

\subsection{Simulation Results}

We analyze two aspects of the CAR-based schemes: feasibility and performance. For feasibility, we have analyzed the delays to form the routing network (HiNet for $\mathrm{CAR}, \mathrm{CAR}+$, and $\mathrm{CAR}++$ and dynamic route creation in MCAR), discover the conzone, and destroy it. The delays for HiNet formation, conzone discovery, and destruction tend to decrease as the number of hops from the critical area to the sink decreases. HiNet formation delay stays under 11 seconds at the maximum and decreases as the transmission range increases. The conzone discovery and destruction delays were found to be less than 1 second. Since CAR is meant for static networks with comparatively long-lived HP streams, these delays are small compared to the duration of HP data flood. In low-mobility scenarios, the delay required for some nodes to refresh their parents and children will be less than the HiNet formation delay, and the delay for nodes that move from off-conzone areas to the conzone to switch to the conzone mode will be small as well.

In our simulations for CAR, this flood duration is set to 50 seconds. CAR is hence a feasible solution that can quickly adjust to different events that require the rediscovery of the conzone. MCAR incurs no HiNet formation delay but incurs a delay in finding a route at the start of HP transmissions. These delays depend on the number of hops but, in our tests, were consistently less than 1 second.

In the following, we present results comparing the performance of our schemes for varying ranges, varying LP data rates, and varying HP data rates.

\subsubsection{Varying Transmission Range}

In this group of simulations, the transmission ranges were varied between 90, 130, 170, and $210 \mathrm{~m}$. As the transmission range increases, the number of hops from the edge of the network to the sink decreases from 6 to 3. The LP data rate of each node, other than the critical area nodes and the sinks, was set to $0.5 \mathrm{pps}$, while the HP data rate of critical area nodes was set to 30 pps. These simulations show the gains of CAR schemes and MCAR as the node density of a deployment increases.

We first make some general observations on the behavioral differences between $\mathrm{CAR}$ and $\mathrm{AODV}+\mathrm{PQ}$. Priority queues provide better service to HP data compared to AODV. However, because each node makes the best decision locally, such a scheme may not be able to provide better service globally. Consider the case in which a node has an empty 


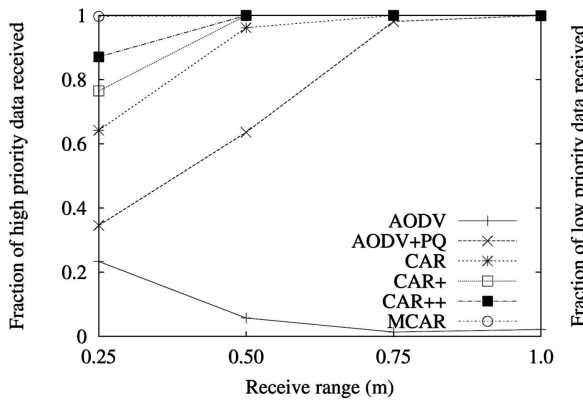

(a)

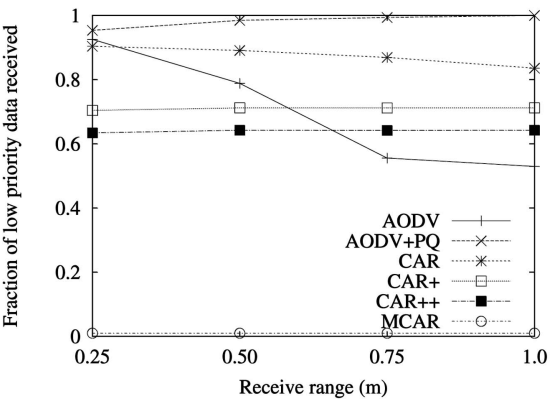

(b)

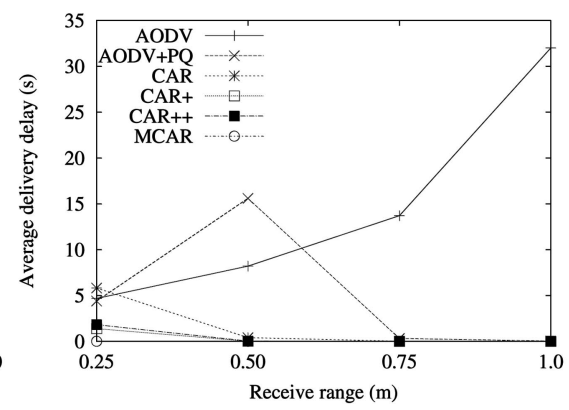

(c)

Fig. 9. Varying transmission range, LP data rate $=0.5 \mathrm{pps}$, and HP data rate $=30$ pps. (a) HP data delivery fraction. (b) LP data delivery fraction. (c) HP data delivery delay.

HP queue but a nonempty LP queue. This node will start injecting LP traffic into the network, which, due to the shared medium, may degrade the service provided to HP packets in nearby nodes. CAR and its enhancements, on the other hand, separate the traffic into two regions and hence eliminate most of the interference that can be caused by having both LP and HP traffic routed on the same paths.

Fig. 9a plots the fraction of HP data delivered to the sink. As the transmission range increases, the network becomes more congested, and more collisions occur. As a result, the performance of AODV degrades severely, and it routes less than 10 percent of HP data successfully. On the other hand, $\mathrm{AODV}+\mathrm{PQ}$ and $\mathrm{CAR}$-based schemes route a higher fraction of the data, although CAR-based schemes route more $\mathrm{HP}$ data than AODV $+\mathrm{PQ}$ for all ranges. At ranges larger than or equal to $130 \mathrm{~m}$, CAR-based schemes route more than 90 percent of the data. We note that $\mathrm{CAR}++$ routes more data than CAR+, which, in turn, routes more data than CAR. Finally, MCAR routes nearly all of the HP data, as it uses MAC-layer mechanisms to silence the conzone and its neighborhood in terms of LP traffic.

Fig. 9b shows the fraction of LP data routed successfully. Although our focus is to provide better service to HP data in the presence of congestion, CAR also effectively utilizes the uncongested off-conzone nodes to prevent severe degradation of LP data. Hence, in addition to improving HP delivery, CAR also enhances delivery of LP traffic as the range increases. The AODV delivery ratio decreases sharply as the range increases, while AODV $+\mathrm{PQ}$ routes the highest percentage of LP data. Note that since AODV $+\mathrm{PQ}$ routes less HP data (see Fig. 9a) and more LP data than CAR-based schemes, it is clear that priority-queue-based schemes alone are not sufficient to provide better service to critical data.

CAR routes more LP data than AODV as the range increases, since it prevents LP data from entering the conzone and getting dropped. AODV $+\mathrm{PQ}$ routes more LP data than CAR, because it does not as aggressively degrade service to LP data as CAR. At large ranges (i.e., in networks with few hops from the sink to the critical area) $\mathrm{AODV}+\mathrm{PQ}$ routes more LP data and approximately the same amount of HP data as CAR. This is because in CAR, congestion may occur in off-conzone areas, as LP data from the conzone is routed out into such areas. Note that CAR+ and CAR++ deliver less LP data compared to CAR, because they, by design, drop more such data.

MCAR drops virtually all LP data. This is due to the close proximity of the LP sinks to the HP source.
MCAR silences all nodes in the communication range of the HP stream; therefore, the LP sink is also silenced. Later in this section, the performance of MCAR in a wider network is presented, and only LP traffic in the communication range of the HP flows is dropped.

Fig. 9c shows that as the range increases, the average HP data delivery delay for AODV increases while such delay for AODV+PQ and CAR-based schemes decreases. This is due to the increasing congestion that AODV faces. Furthermore, the jitter introduced by data forwarding is always less for CAR-based schemes as compared to AODV and AODV $+\mathrm{PQ}$ (not shown).

In our simulations, we observed that despite shorter paths being available, AODV and AODV + PQ do not necessarily route data along such paths. MCAR also suffers from this problem. CAR, CAR+, and CAR++ schemes always find a shorter path. Routing along shortest paths has several implications, including less overall network energy usage. Since MCAR uses AODV's route discovery algorithm and does not use the HiNet, it also sometimes finds suboptimal paths to the sink.

Fig. 10 shows the maximum energy used by any node in the deployment. This includes the energy used to route all possible traffic, both LP and HP. The energy used by AODV and $\mathrm{AODV}+\mathrm{PQ}$ is more than that for CAR. CAR+ uses less energy, and CAR++ uses the minimum energy among all the schemes. MCAR falls in between: it saves energy over AODV by reducing the amount of LP contention during HP traffic times; however, MCAR routes may contain extra hops as compared to CAR, because it does not benefit from the HiNet creation phase.

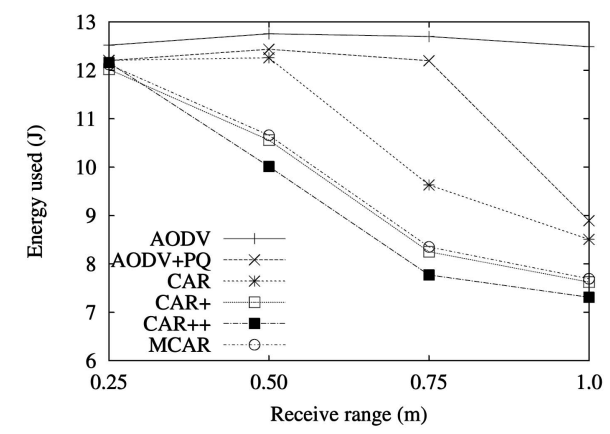

Fig. 10. Maximum node energy used: varying transmission range, $\mathrm{LP}$ data rate $=0.5 \mathrm{pps}$, and $\mathrm{HP}$ data rate $=30 \mathrm{pps}$. 


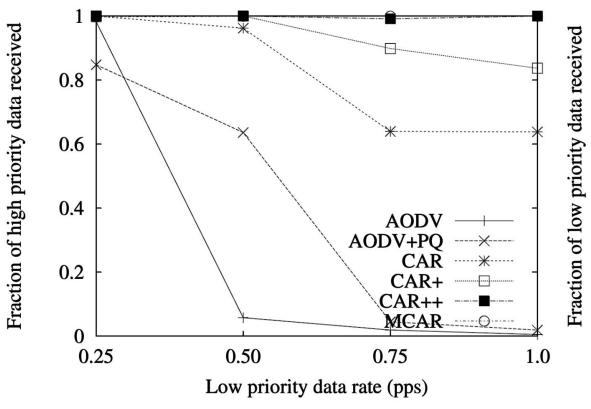

(a)

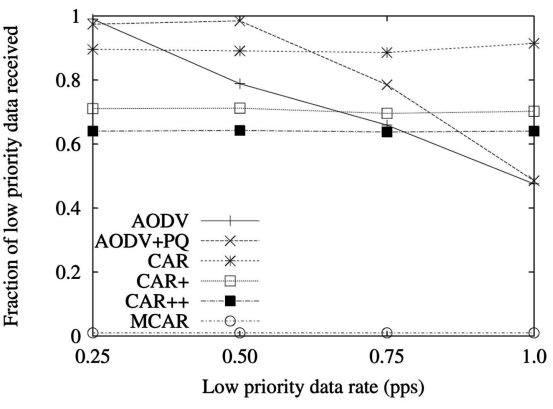

(b)

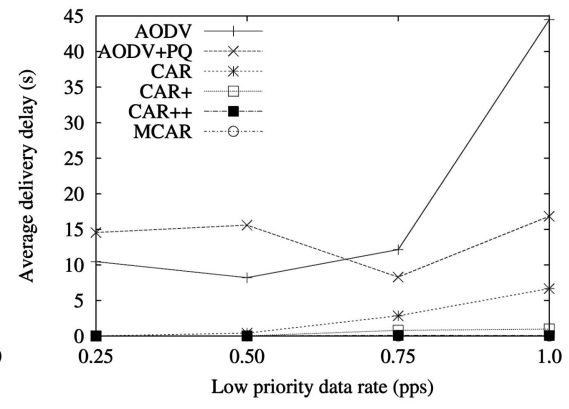

(c)

Fig. 11. Varying LP data rate, transmission range $=130 \mathrm{~m}$, and HP data rate $=30 \mathrm{pps}$. (a) HP data delivery fraction. (b) LP data delivery fraction. (c) HP data delivery delay.

\subsubsection{Varying Low Priority Data Rate}

In this set of simulations, the range is set to $130 \mathrm{~m}$, and the $\mathrm{HP}$ data rate of each critical area node is set to $30 \mathrm{pps}$, while the LP data rate is varied. These simulations compare the performance of CAR schemes and MCAR with AODV and $\mathrm{AODV}+\mathrm{PQ}$ as the network has to contend with increasingly intense background LP traffic.

As the LP data rate increases, the fraction of HP packets routed by AODV sharply falls to zero (see Fig. 11a). Although AODV $+\mathrm{PQ}$ performs better than AODV, it still faces the same fate. In contrast, though the fraction of data routed successfully to the sink by the CAR-based schemes decreases, these schemes still route more than 60 percent of the data, even when AODV and AODV+PQ do not route any data at all. MCAR is less sensitive to the LP data rate, since it silences all nodes within the communication range of the HP flows. Note that the curve for CAR++ overlaps the curve for MCAR in Figs. 11a and 11c.

Also, the fraction of LP data successfully routed by AODV drops (see Fig. 11b). For CAR, it decreases from around 90 percent to 85 percent. For CAR+ and CAR++, this fraction stays almost constant. MCAR still drops nearly all of the LP data due to the close proximity of the LP sinks to the HP sink.

The delays for AODV and CAR increase, while they stay almost constant for CAR+, CAR++, and MCAR (see Fig. 11c). The standard deviations of the delivery delays (not shown) exhibit higher variations for AODV and $\mathrm{AODV}+\mathrm{PQ}$. Such high variations correspond to larger jitter values, which are a problem for real-time data delivery.
Since AODV routes a very small fraction of HP packets and a smaller fraction of LP packets, the maximum energy used by AODV stays the same as the rate of LP data varies (not shown). The energy consumed in the CAR-based schemes increases as the LP data rate becomes larger. In all cases, it is lower than AODV.

\subsubsection{Varying High Priority Data Rate}

In the final set of simulations, the HP data rate was varied, and the LP data rate was fixed to $0.5 \mathrm{pps}$. The communication range was fixed at $130 \mathrm{~m}$. These simulations compare the performance of CAR schemes and MCAR with AODV and $\mathrm{AODV}+\mathrm{PQ}$ for $\mathrm{HP}$ data rates ranging from the same as the LP data rate of 0.5-30 pps.

As shown in Fig. 12a, though the fraction of HP data successfully delivered is 100 percent for all schemes when the HP data rate is low, as it increases, the fraction of $\mathrm{HP}$ data routed decreases faster for AODV and AODV+PQ than for CAR-based schemes. Again, MCAR is insensitive to the increase, as long as the total HP traffic level remains below the network capacity. Note that the curve for CAR++ overlaps the curves for CAR+ and MCAR in Figs. 12a and 12c. Fig. 12b depicts the LP data delivery fraction as the HP data rate was varied. These results are consistent with those already presented.

Though the average delivery delay increases for all schemes, as shown in Fig. 12c, MCAR has the smallest delay. As the HP data rate increases, AODV and $\mathrm{AODV}+\mathrm{PQ}$ have higher variation in delivery delay as compared to CAR-based schemes (not shown). The

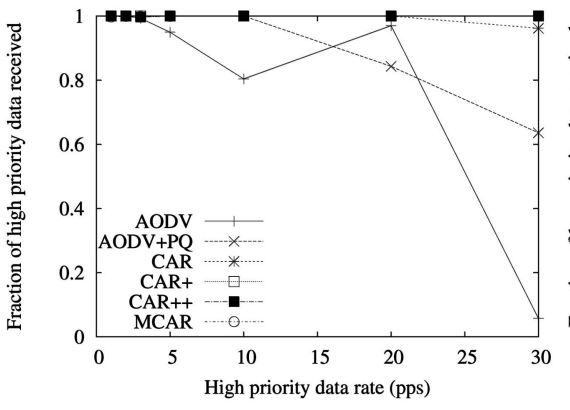

(a)

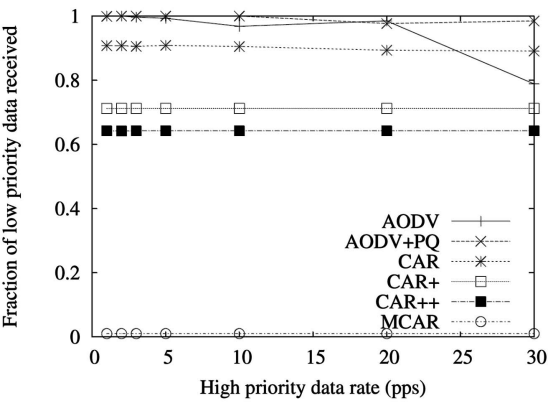

(b)

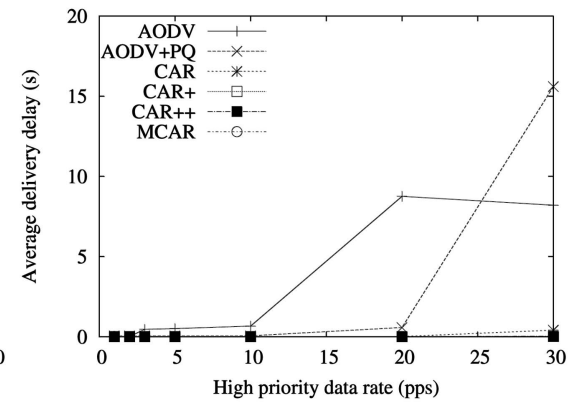

(c)

Fig. 12. Varying HP data rate, transmission range $=130 \mathrm{~m}$, and LP data rate $=0.5$ pps. (a) HP data delivery fraction. (b) LP data delivery fraction. (c) HP data delivery delay. 


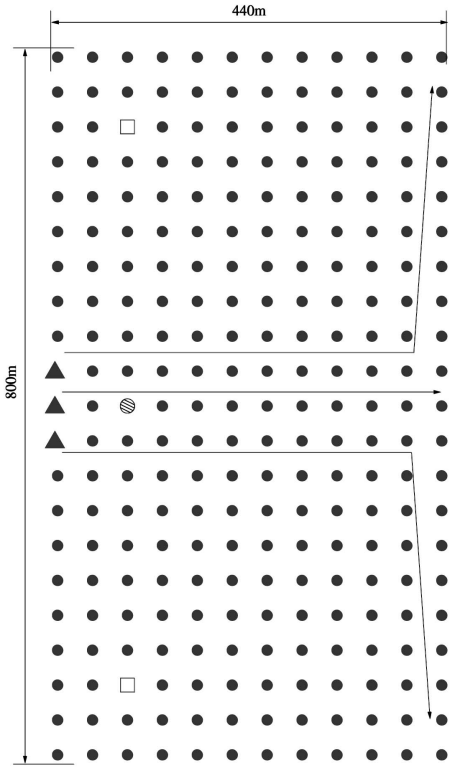

Fig. 13. Wide network deployment for static and mobile experiments.

CAR variants also provide even energy consumption as compared with AODV and AODV+PQ (not shown).

\subsection{MAC-Enhanced Congestion Aware Routing in Wider Networks}

Since MCAR requires nodes within the communication range of the HP flows to drop all LP data, in narrow networks such as the one tested in the previous simulations, a large portion of LP data is suppressed. This is in contrast with CAR, which delivers much more LP data than MCAR. MCAR makes this trade-off to ensure that the largest possible percentage of HP data is routed successfully through the network. The performance of CAR in a wider network with the same amount of HP traffic will be the same in terms of the HP data delivery and better in terms of LP data delivery due to the absence of congestion in a larger part of the network. Since CAR delivers some LP data, even in such narrow networks, we now explore the performance of MCAR for LP data delivery in wider networks. Note that CAR does not require the critical area to be on a horizontal line from the sink. The simulation setup used in this paper uses such critical areas for the simplicity of presentation. CAR's component algorithms can handle critical areas located anywhere in the network and not necessarily on a horizontal line with the HP sink.

To demonstrate that MCAR does not silence the entire network, tests were run on a static network 12 nodes deep and 21 nodes wide (see Fig. 13). HP sources are placed in the middle of the long edge and route data across to a sink on the opposite wide edge. LP sinks are in two corners of the network, with all other nodes generating LP traffic destined for one of the two LP sinks at a rate of $0.5 \mathrm{pps}$. The $\mathrm{HP}$ nodes generate traffic at a rate of 30 pps.

As expected, the center of the network is reserved for HP flows, but nodes outside the communication range of those flows are free to transfer LP data. As the communication range is increased, a lower percentage of LP data is received due to an increase in the number of nodes in the shadow mode. However, at all communication ranges, MCAR delivers more than 90 percent of the HP data and 45 percent and 65 percent of the LP traffic for all but the largest transmission ranges (see Fig. 14a).

\subsection{Dynamic High Priority Sources}

Here, we present simulations to demonstrate the ability of MCAR to deliver HP packets when the sources of the HP data are dynamic. Additionally, these experiments show that LP flows recover rapidly after HP streams end.

To demonstrate the flow setup and tear down speed, we use the 250-node network configuration in Section 4.3. Fig. 14b shows the number of HP and LP packets received per second of simulation. The three HP sources generate data at $30 \mathrm{pps}$ for 10 seconds and then go silent for 10 seconds. Fig. 14c shows the same information, with HP sources generating packets at $30 \mathrm{pps}$ for 1 second and then going silent for 9 seconds. LP data reception rapidly decreases during times when the HP sources generate data and then increases rapidly directly after those sources go silent. Longer burst times for HP data, of course, suppress a greater number of LP packets. This periodic behavior is exactly the goal of the design of MCAR. HP packets get use of the paths that they need and suppress LP traffic only along those paths and only during the times when HP data is present in the network. The overall delivery percentages for HP and LP data are similar to those of the previous sections.

Finally, to show that MCAR supports mobility effectively, simulations are run with the network configuration depicted in Fig. 13 with mobile HP sources. At the start of the simulation, all nodes, except the sinks and the HP sources,

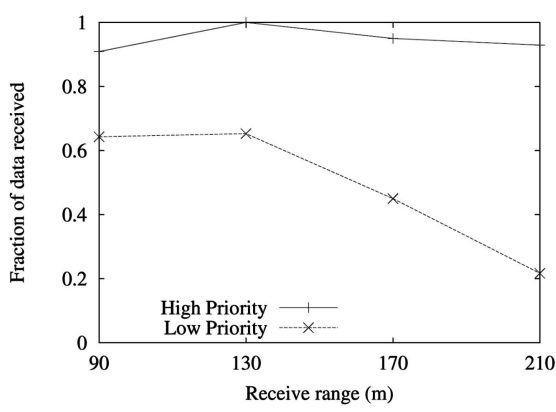

(a)

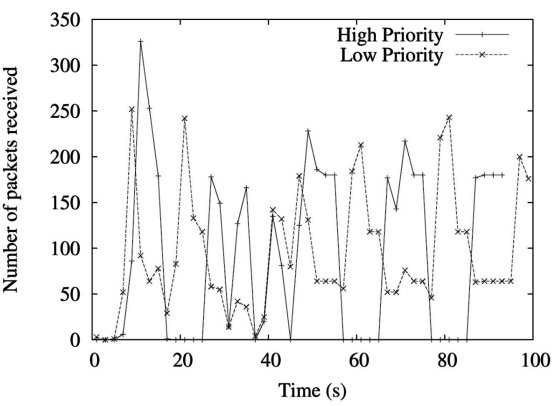

(b)

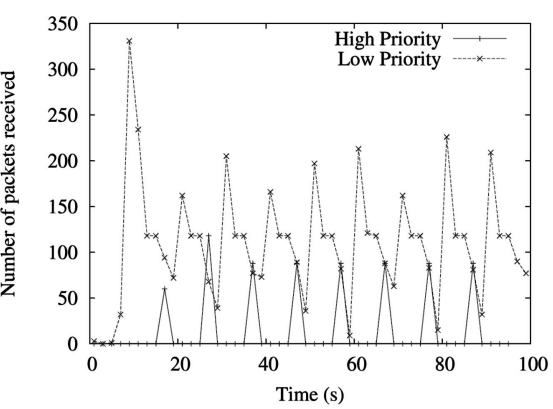

(c)

Fig. 14. Fraction of HP and LP packets received. (a) Fraction of HP and LP data received, with varying transmission range. (b) HP and LP packets received, with 0.5 duty cycle. (c) HP and LP packets received, with 0.1 duty cycle. 


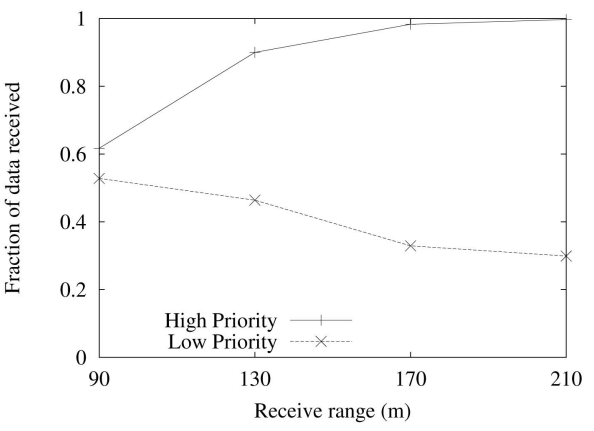

Fig. 15. Mobility: fraction of HP and LP data received.

are sending LP data at a rate of $0.5 \mathrm{pps}$. All three HP sources begin generating data and then begin moving at $8 \mathrm{~m} / \mathrm{s}$ toward the sink for 60 seconds. Then, the middle HP node stops moving, and the other two nodes turn toward the edges of the network and move away from the HP sink.

With a short reception range, MCAR delivers over 60 percent of the HP packets. Most of the HP loss is due to route changes because of mobility (see Fig. 15). Again, as the reception range increases, the percentage of HP packets received at the source increases rapidly. Finally, LP nodes outside the communication range of the HP traffic still successfully deliver LP packets.

\section{Implementation Case Study}

To demonstrate the practicality of our algorithms and to verify the conclusions drawn from our simulations, we implemented MCAR on our sensor network testbed.

Our testbed is deployed in a $10 \mathrm{~m} \times 11 \mathrm{~m}$ laboratory in a grid suspended $0.6 \mathrm{~m}$ from the ceiling and $2.4 \mathrm{~m}$ above the floor. The network is made up of 48 EyesIFXv2 nodes [11], separated by $1.6 \mathrm{~m}$ in one direction and $1.2 \mathrm{~m}$ in the other direction.

The EyesIFXv2 nodes were developed during a 3-year European research project on self-organizing energy-efficient sensor networks [11]. The nodes use an ultra-lowpower MSP430 processor with a 10-Kbyte on-chip RAM, a 48-Kbyte flash/ROM, and an additional a 512-Kbyte serial EPROM. The radio chip is a low-power FSK/ASK transceiver, providing half-duplex low-data-rate communication in the $868-\mathrm{MHz}$ ISM band. It operates using FSK modulation, with a sensitivity of $<-109 \mathrm{dBm}$, enabling half-duplex wireless connectivity of up to 64 kilobits per second (Kbps). The radio chip provides a digital potentiometer to allow the transmission power and, hence, the transmission range of a node to be adjusted. This allows the testbed to range from one to five hops from corner to corner. A USB backplane is used to monitor the network without interfering with the flow of data.

The EyesIFX platform is equipped with a temperature and a light intensity sensor. Also, the TDA5250 chip provides an RSS indicator via both an internal register that can be queried or an analog pin, whose Vcc varies from $0 \mathrm{mV}$ (silence) up to $1,350 \mathrm{mV}$, linked to the ADC of the MSP430. A minimum RSSI threshold can be set to consider the received power to be valid data.

\subsection{Environmental Monitoring with MAC-Enhanced Congestion Aware Routing}

We chose MCAR to demonstrate that the differentiated routing algorithms and MAC-layer enhancements in MCAR used for mobility support can be implemented on the current hardware. MCAR is also suited for our particular application, since the causes of changes in environmental conditions (e.g., fires) would be expected to spread and therefore be a potentially mobile source of data.

To put MCAR to use, an environmental monitoring application that tracks temperature readings throughout the sensor network was augmented. Our application monitors the environment, periodically sending temperature readings (LP data) from every node to a sink at a low rate (one every few seconds). In the event that the temperature increases beyond a threshold, events are generated at a higher rate (HP data), and alarms are triggered at the sink. Additionally, nodes send information about the ambient light levels periodically (LP data). To ensure that messages are delivered to the sink in the face of node failure(s), the application uses a very simple flooding mechanism. One major challenge in the system is that nodes rapidly became congested with traffic, and often, when an alarm should be triggered, the relevant data never reach the sink.

The testbed periodically simulates a temperature alarm by telling a node that its temperature is above the alarm threshold, and this alarm is transmitted to the HP sink. Alarm messages are transmitted at a frequency five times higher than that of the LP polling messages. The HP sink is in a corner of the network, and the data is delivered to it through a corridor. In this corridor, flooding is being used to propagate LP data. In case of a fire alarm, it is important to deliver the messages as soon as possible, and a static route would be inappropriate, because nodes in the congested region of the network drop far too many packets; however, routing around the region is also impossible.

We also test MCAR's ability to track moving sources by having the testbed simulate temperature alarms that traverse the edge of the network. Static HP routes such as those built by CAR will not work for varying locations of the temperature alarm, allowing the demonstration of MCAR's flexibility.

We implemented the applications described above and the MCAR combined MAC and routing algorithms with its modified RTS/CTS described in Section 3.3 in TinyOS 1 [9]. The environmental monitoring application has a timer that runs periodically. When the timer fires, the battery, light, and temperature sensors are polled, and the measurements are converted to digital values. If the temperature reading is above a user-defined threshold, which can be adjusted interactively by users via commands sent over the radio or USB backbone, the node transitions into the HP mode. Finally, the send procedure is called with the appropriate priority setting.

The size of the whole application is 22 Kbytes, which can completely reside in the ROM of the sensor node. Most of the size of the application is related to the RF components and hardware management, and the MCAR modifications amount to less than 100 bytes.

The primary challenge in implementing MCAR involved the strictly modular design of TinyOS. Because MCAR relies on priority information from the application layer and 


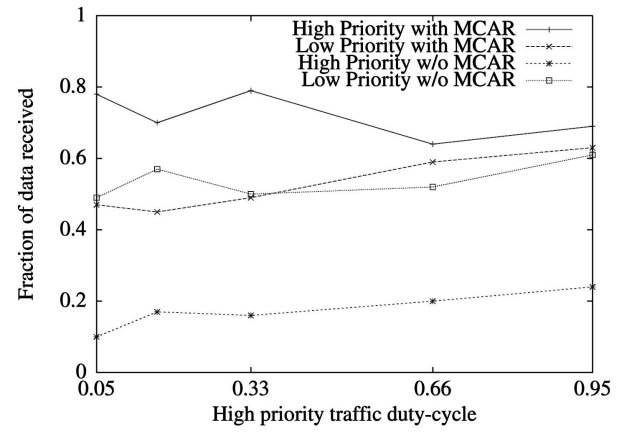

Fig. 16. Testbed: fraction of HP and LP data received versus HP traffic duty cycle.

alters both the routing and MAC layers, it was necessary to find clean ways to pass information between the layers. Additionally, the purely event-driven nature required a state-machine-style algorithm design. Fortunately, because MCAR's mechanisms work in a top-down manner (i.e., the adaptations are driven by the application priority settings), only these priorities need to be exposed to all layers. For example, any route setup packets for an HP flow must be assigned an HP, or they risk being dropped. However, route setup in many standard protocols is not tagged with flow information. Therefore, application priorities must be used at the routing layer, and all routing mechanisms used to service an HP flow must themselves be HP. While such changes in protocols are small, in terms of code size, they are critical for protocol correctness.

\subsection{Testbed Results}

In this section, we present results from running our application enhanced with MCAR. The network is configured with an HP sink on one edge of the network and LP sinks at two corners of the network. All nodes, except the sinks, run the monitoring application. A source is chosen as the trigger of an alarm.

The first test performed involves varying the HP duty cycle in the range of $[0.05,0.95]$, keeping the HP data rate at a constant 5 pps and keeping the LP data rate at a constant 0.33 pps from each node. Fig. 16 shows that MCAR maintains an HP delivery rate above 90 percent while the LP data delivery rate varies between 47 percent and 60 percent. The reason that the fraction of LP data received increases as the HP duty cycle increases is that since many LP nodes are shadowed, there is less LP contention in the network due to the flooding algorithm. Therefore, congestion at the LP sinks decreases.

We also test the setup and recovery time of HP flows by choosing a node at the edge of the network and moving its temperature threshold down to trigger HP events at duty cycles of $0.05,0.33$, and 0.66 , representing increasingly longer HP flows in the network. Figs. 17a, 17b, and 17c depict the number of HP and LP packets received in each 3-second interval. As can be seen, the decrease in LP packets received corresponds directly to the HP traffic, and the fraction of LP packets received increases soon after the HP traffic stops.

Finally, we test the ability of MCAR to continue delivering $\mathrm{HP}$ packets in the face of mobility. For these tests, the HP duty cycle was kept in the range of $[0.05,0.95]$, the HP data rate was set at a constant 5 pps, and the LP data rate was set at a constant 0.33 pps from each node. A heating event was moved through the network, following the mobility pattern in the simulation (see Fig. 13). Fig. 18 shows that MCAR maintains an HP delivery rate above 78 percent for all but the lowest transmit power setting. Without MCAR active, the HP traffic suffers heavy losses due to the congestion in the network.

\section{CONCLUSion AND FutURe Work}

In this paper, we addressed data delivery issues in the presence of congestion in wireless sensor networks. We proposed CAR, which is a differentiated routing protocol and uses data prioritization. We also develop MCAR, which deals with mobility and dynamics in the sources of HP data.

Our extensive simulations show that as compared to AODV and AODV $+P Q, C A R$ and its variants increase the fraction of HP data delivery and decrease delay and jitter for such delivery while using energy more uniformly in the deployment. CAR also routes an appreciable amount of LP data in the presence of congestion. We additionally show that MCAR maintains HP data delivery rates in the presence of mobility and show that the route setup and tear-down times associated with the HP flows are minimal.

Both CAR and MCAR support effective HP data delivery in the presence of congestion. CAR is better suited for static networks with long-duration HP floods. For bursty $\mathrm{HP}$ traffic and/or mobile HP sources, MCAR is a better fit.

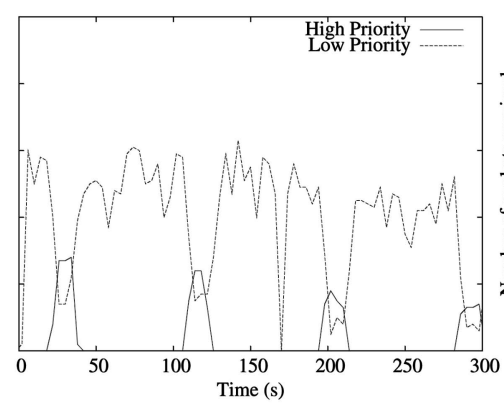

(a)

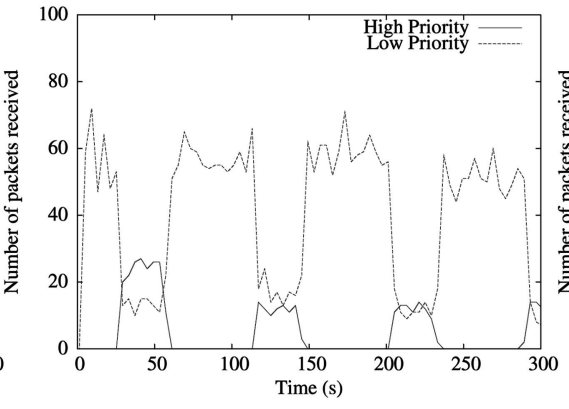

(b)

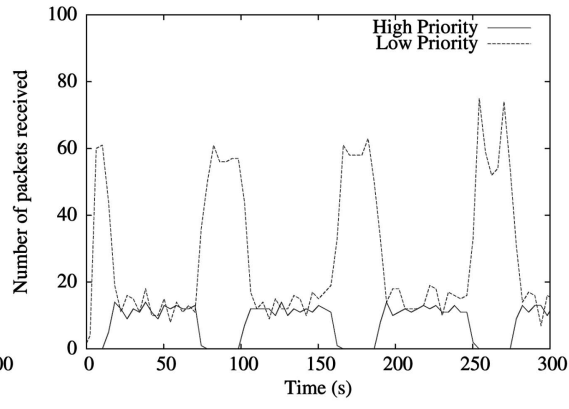

(c)

Fig. 17. Testbed: HP and LP packets received. (a) Duty cycle of 0.05 . (b) Duty cycle of 0.33 . (c) Duty cycle of 0.66 . 


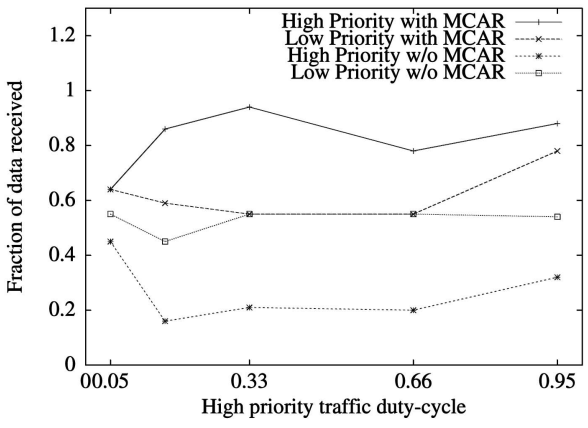

Fig. 18. Testbed with mobility: fraction of HP and LP data received versus HP traffic duty cycle.

We also presented the implementation of an environmental monitoring system that uses MCAR as its MAC and routing layer. Our experiments on the testbed verify the conclusions drawn from the simulation study and show that MCAR is suitable for implementation on currently available hardware.

Because of the low jitter rates and maintainable delay, CAR and its variants appear suitable to real-time data delivery. To ensure QoS for video streams, reactive dropping methods could be combined into the routing protocol. Our future work looks at the effectiveness of such techniques in sensor network environments. Also, while MCAR merges multiple conzones naturally, we are now exploring the interactions of differentiated routing and multiple conzones, which may be overlapping or disjoint in CAR and its two enhancements. Finally, we will also explore the impact of different sizes and shapes of conzones on data delivery in the future.

\section{ACKNOWLEDGMENTS}

The authors would like to thank Farooq Anjum of Telcordia Technologies for insightful discussions. This research was sponsored in part by the US Army Research Laboratory and the UK Ministry of Defence and was accomplished under Agreement W911NF-06-3-0001 and by the US National Science Foundation under Grant CNS0519460. This work was conducted when Riccardo Crepaldi and Albert F. Harris III were with Department of Information Engineering, University of Padova.

\section{REFERENCES}

[1] Draft Supplement to Part 11: Wireless Medium Access Control (MAC) and Physical Layer (PHY) Specifications: Medium Access Control (MAC) Enhancements for Quality of Service (QoS), IEEE 802.11e/ D4.0, Nov. 2002.

[2] G.-S. Ahn, S.G. Hong, E. Miluzzo, A.T. Campbell, and F. Cuomo, "Funneling-MAC: A Localized, Sink-Oriented MAC for Boosting Fidelity in Sensor Networks," Proc. Fourth ACM Conf. Embedded Networked Sensor Systems (SenSys), 2006.

[3] G.-S. Ahn, L.-H. Sun, A. Veres, and A.T. Campbell, "Swan: Service Differentiation in Stateless Wireless Ad Hoc Networks," Proc. IEEE INFOCOM, 2002.

[4] K. Akkaya and M.F. Younis, "An Energy-Aware QoS Routing Protocol for Wireless Sensor Networks," Proc. 23rd IEEE Int'l Conf. Distributed Computing Systems (ICDCS '03), pp. 710-715, 2003.

[5] S.R. Das, C.E. Perkins, and E.M. Belding-Royer, "Performance Comparison of Two On-Demand Routing Protocols for Ad Hoc Networks," Proc. IEEE INFOCOM '00, pp. 3-12, 2000.
[6] C.T. Ee and R. Bajcsy, "Congestion Control and Fairness for Many-to-One Routing in Sensor Networks," Proc. Second ACM Conf. Embedded Networked Sensor Systems (SenSys '04), pp. 148-161, 2004.

[7] E. Felemban, C.-G. Lee, and E. Ekici, "MMSPEED: Multipath Multi-SPEED Protocol for QoS Guarantee of Reliability and Timeliness in Wireless Sensor Networks," IEEE Trans. Mobile Computing, vol. 6, pp. 738-754, 2006.

[8] T. He, J.A. Stankovic, C. Lu, and T. Abdelzaher, "Speed: A Stateless Protocol for Real-Time Communication in Sensor Networks," Proc. 23rd IEEE Int'l Conf. Distributed Computing Systems (ICDCS), 2003.

[9] J. Hill, R. Szewczyk, A. Woo, S. Hollar, D. Culler, and K. Pister, "System Architecture Directions for Network Sensors," Proc. Ninth Int'l Conf. Architectural Support for Programming Languages and Operating Systems (ASPLOS '00), Nov. 2000.

[10] B. Hull, K. Jamieson, and H. Balakrishnan, "Mitigating Congestion in Wireless Sensor Networks," Proc. Second ACM Conf. Embedded Networked Sensor Systems (SenSys), 2004.

[11] Eyesifxv2 Version 2.0. Infineon, http://www.infineon.com, 2008.

[12] C. Intanagonwiwat, R. Govindan, and D. Estrin, "Directed Diffusion: A Scalable and Robust Communication Paradigm for Sensor Networks," Proc. ACM MobiCom '00, Aug. 2000.

[13] D.B. Johnson and D.A. Maltz, "Dynamic Source Routing in Ad Hoc Wireless Networks," Mobile Computing, pp. 153-181, Kluwer Academic Publishers, Feb. 1996.

[14] B. Karp and H. Kung, "GPSR: Greedy Perimeter Stateless Routing for Wireless Networks," Proc. ACM MobiCom, 2000.

[15] C. Lu, B. Blum, T. Abdelzaher, J. Stankovic, and T. He, "RAP: A Real-Time Communication Architecture for Large-Scale Wireless Sensor Networks," Proc. Eighth IEEE Real-Time and Embedded Technology and Applications Symp. (RTAS '02), pp. 55-66, 2002.

[16] S. Madden, M. Franklin, J. Hellerstein, and W. Hong, “Tag: A Tiny Aggregation Service for Ad-Hoc Sensor Networks," Proc. Fifth Symp. Operating System Design and Implementation (OSDI), 2002.

[17] ns2: Network Simulator, http://www.isi.edu/nsnam/ns/, 2008.

[18] C.E. Perkins and E.M. Royer, "Ad Hoc On-Demand Distance Vector Routing," Proc. Second IEEE Workshop Mobile Computing Systems and Applications (WMCSA '99), Feb. 1999.

[19] J. Polastre, J. Hill, and D. Culler, "Versatile Low Power Media Access for Wireless Sensor Networks," Proc. Second ACM Conf. Embedded Networked Sensor Systems (SenSys), 2004.

[20] S. Rangwala, R. Gummadi, R. Govindan, and K. Psounis, "Interference-Aware Fair Rate Control in Wireless Sensor Networks," Proc. ACM SIGCOMM, 2006.

[21] N. Shrivastava, C. Buragohain, D. Agrawal, and S. Suri, "Medians and Beyond: New Aggregation Techniques for Sensor Networks," Proc. Second ACM Conf. Embedded Networked Sensor Systems (SenSys), 2004.

[22] C.-Y. Wan, S.B. Eisenman, and A.T. Campbell, "CODA: Congestion Detection and Avoidance in Sensor Networks," Proc. First ACM Conf. Embedded Networked Sensor Systems (SenSys '03), pp. 266-279, 2003.

[23] A. Woo and D.E. Culler, "A Transmission Control Scheme for Media Access in Sensor Networks," Proc. ACM MobiCom, 2001.

[24] W. Ye, J. Heidemann, and D. Estrin, "An Energy-Efficient MAC Protocol for Wireless Sensor Networks," Proc. IEEE INFOCOM, 2002.

[25] H. Zhang, A. Arora, Y. Choi, and M. Gouda, "Reliable Bursty Convergecast in Wireless Sensor Networks," Proc. ACM MobiHoc, 2005.

[26] Y. Zhang, M.P.J. Fromherz, and L.D. Kuhn, "Smart Routing with Learning-Based QoS-Aware Meta-Strategies," Proc. First Workshop Quality of Service Routing (WQoSR '04), pp. 298-307, 2004.

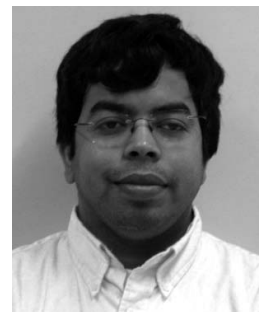

Raju Kumar received the BTech degree in computer science and engineering from the Indian Institute of Technology, Kanpur, in 2003. $\mathrm{He}$ is currently working toward the $\mathrm{PhD}$ degree in the Department of Computer Science and Engineering, The Pennsylvania State University, where he is also a member of the Networking and Security Research Center. His research interests include ad hoc, sensor, and mesh networks. He is a student member of the IEEE. 


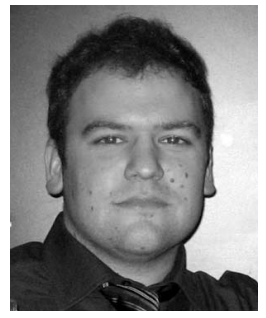

Riccardo Crepaldi received the MS (Laurea) degree in telecommunications engineering from the University of Padova, Padova, Italy, in 2006. After his graduation, he was a research scientist with the Signet Group, University of Padova, until 2007. His research focused on wireless sensor networks (WSNs), and he designed and deployed a WSN testbed and developed management tools for it. He also worked on the design and performance analysis of routing and localization algorithms for WSNs. He is currently working toward the $\mathrm{PhD}$ degree in the Department of Computer Science, University of Illinois, Urbana-Champaign, under the supervision of Professor Robin Kravets. His research interests include systems design and management and service discovery for wireless sensor networks and delay tolerant networks. He is a student member of the IEEE.

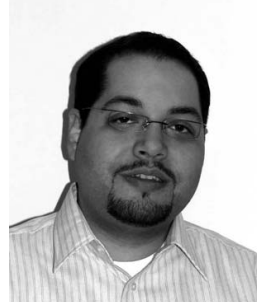

Hosam Rowaihy received the BS degree in computer engineering from King Fahd University of Petroleum and Minerals, Dhahran, Saudi Arabia, and the MS degree in electrical engineering from the University of Maryland, College Park. He is currently working toward the PhD degree in the Department of Computer Science and Engineering, The Pennsylvania State University, where he is also a member of the Networking and Security Research Center. His research interests include resource management in sensor networks, peer-to-peer networks, and RFID systems. He is a student member of the IEEE.

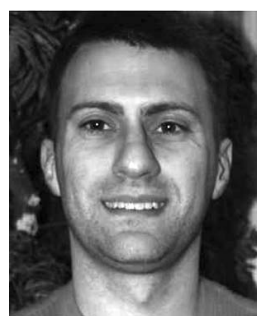

Albert F. Harris III received the $\mathrm{PhD}$ degree in computer science from the University of Illinois, Urbana-Champaign, specializing in wireless network protocol and mobile systems design. $\mathrm{He}$ is currently a research assistant professor in the Center for Remote Sensing of Ice Sheets (CReSIS), University of Kansas. He has led computer science research on the US-Armyfunded projects for both Global Information Systems Technology, Inc. and CPResearch, Inc. Prior to graduate school, he operated a computer network consulting business in Kansas City, Missouri, for more than five years. $\mathrm{He}$ is a member of the IEEE.

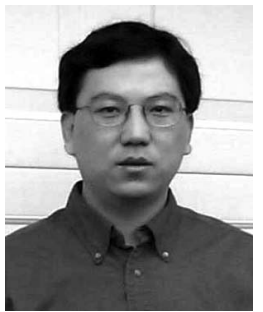

Guohong Cao received the BS degree from Xian Jiaotong University, Xian, China, and the MS and PhD degrees in computer science from the Ohio State University in 1997 and 1999, respectively. Since then, he has been with the Department of Computer Science and Engineering, The Pennsylvania State University, where he is currently an associate professor. His research interests include wireless networks and mobile computing. He has published more than 100 papers in sensor networks, wireless network security, data dissemination, resource management, and distributed fault-tolerant computing. He has served on the editorial boards of the IEEE Transactions on Mobile Computing and the IEEE Transactions on Wireless Communications and on the program committee of many conferences. He was a recipient of a US National Science Foundation Faculty Early Career Development (CAREER) Award in 2001. He is a senior member of the IEEE and the IEEE Computer Society.

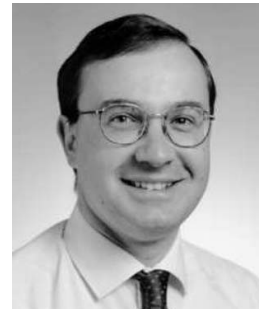

Michele Zorzi received the Laurea and $\mathrm{PhD}$ degrees in electrical engineering from the University of Padova, Padova, Italy, in 1990 and 1994, respectively. During the academic year 1992-1993, he was on leave from the University of California, San Diego (UCSD), attending graduate courses and doing research on multiple access in mobile radio networks. In 1993, he joined the faculty of the Dipartimento di Elettronica e Informazione, Politecnico di Milano, Milano, Italy. After spending three years with the Center for Wireless Communications, UCSD, in 1998, he joined the School of Engineering, University of Ferrara, Ferrara, Italy. In 2003, he joined the Department of Information Engineering, University of Padova, where he is currently a professor. His research interests include performance evaluation in mobile communications systems, random access in mobile radio networks, ad hoc and sensor networks, and energy-constrained communications protocols. From 2003 to 2005, he was the editor in chief of the IEEE Wireless Communications Magazine. He currently serves on the steering committee of the IEEE Transactions on Mobile Computing and is on the editorial boards of the IEEE Transactions on Communications, the IEEE Transactions on Wireless Communications, the Wiley Journal of Wireless Communications and Mobile Computing, and the ACM/URSI/Kluwer Journal of Wireless Networks. He was also guest editor of the IEEE Personal Communications Magazine (special issue on energy management in personal communications systems) and the IEEE Journal on Selected Areas in Communications (special issue on multimedia network radios). He is a fellow of the IEEE.

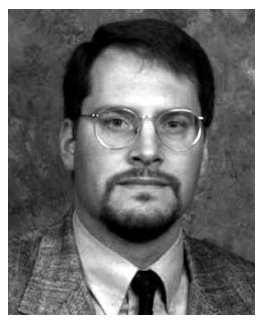

Thomas F. La Porta received the BSEE and MSEE degrees from the Cooper Union, New York, and the PhD degree in electrical engineering from Columbia University, New York. He is a distinguished professor in the Department of Computer Science and Engineering, The Pennsylvania State University (Penn State), where he is also the director of the Networking and Security Research Center. Prior to joining Penn State, he was the director of the Mobile Networking Research Department, Bell Laboratories (Bell Labs), where he led various projects in wireless and mobile networking. He is the founding editor in chief of the IEEE Transactions on Mobile Computing. His research interests include mobility management, signaling and control for wireless networks, mobile data systems, and protocol design. He has published more than 100 technical papers and is the holder of 30 patents. $\mathrm{He}$ is a fellow of the IEEE and the Bell Labs. He received a Thomas Alva Edison Patent Award.

$\triangleright$ For more information on this or any other computing topic, please visit our Digital Library at www.computer.org/publications/dlib. 